\title{
Estudio de la organización de la sismicidad en torno al terremoto del 24 de julio del 2001 en el norte de Chile
}

\author{
Study of the seismic organization around the earthquake of July 24th, \\ 2001 in northern Chile
}

\author{
Bianca Glass $^{1} \quad$ Carlos Leiva $^{1} \quad$ Carlos Meneses $^{1}$ \\ Recibido 26 de marzo de 2012, aceptado 2 de julio de 2013 \\ Received: March 26, 2012 Accepted: July 2, 2013
}

\begin{abstract}
RESUMEN
En este trabajo se analiza la organización de la sismicidad asociada al terremoto del 24 de julio del 2001 en la quebrada de Aroma, en el norte de Chile, utilizando datos provenientes de la red sismológica perteneciente a la Universidad de Tarapacá en Arica, Chile. Se encuentra que hay rasgos interesantes en la organización en una zona definida alrededor del hipocentro. De hecho, se construye un índice fractal que tiene importantes valores estadísticos antes, durante y después del evento, lo que puede arrojar algo de luz sobre la previsibilidad de eventos mayores.
\end{abstract}

Palabras clave: Multifractal, análisis espacial, sismología, organización sísmica, terremoto.

\begin{abstract}
This paper analyses the organization of the seismicity associated with the 24th July 2001 earthquake in "Quebrada de Aroma," northern Chile using data from the seismological network belonging to the Universidad de Tarapacá, Arica, Chile. There are very important features in the organization in a defined zone around the hypocenter. In fact, a fractal index is built, having significant statistical values before, during and after the event, such as that it can shed some light about predictability of major events.
\end{abstract}

Keywords: Multifractal, spatial analysis, seismology, seismic organization, earthquake.

\section{INTRODUCCIÓN}

El 24 de julio de 2001 el terremoto que ocurrió en la quebrada de Aroma, en la decimoquinta región de Chile (Figura 1), provocó muchos daños en los pequeños pueblos cercanos al epicentro. Este terremoto tuvo su hipocentro a una profundidad (h) de $3 \mathrm{~km}$ latitud (La) 19,5 S y longitud (Lo) de 69,3 W, con una magnitud Richter de $\mathrm{Mw}=6,4$ [1] y una intensidad de VII en Chuzmisa, VI en Huara y V en Arica e Iquique. Su hipocentro se localizó en un lugar bajo la quebrada de Aroma, la que pertenece a una zona de fractura estudiada con algún detalle [2-3].

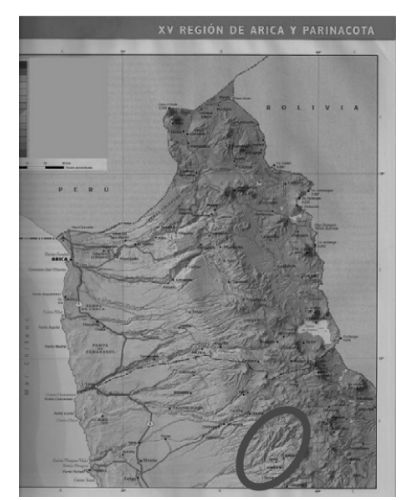

Figura 1. El círculo muestra la quebrada de Aroma.

\footnotetext{
1 Departamento de Física. Facultad de Ciencias. Universidad de Tarapacá. Casilla 7-D. Arica, Chile. E-mail: bglass@uta.cl; cleivas@uta.cl; cmeneses@uta.cl
} 
La zona en que se originó la ruptura ha mostrado mucha actividad en los últimos años y representa riesgo para algunas infraestructuras urbanas y de las redes de conectividad (edificios, puentes, etc.) en la región ubicada entre las ciudades de Iquique y Arica, incluyendo varios pueblos entremedio.

La sismicidad superficial que se observa en el altiplano chileno es, en general, menos frecuente que la numerosa sismicidad asociada al contacto sismogénico interplaca, sin embargo, después del terremoto del 23 de junio del 2001 ocurrido en el sur de Perú se observó un incremento en la actividad sísmica superficial en esta región [4].

La zona estudiada consiste en una fractura de característica vertical que aparentemente responde a las fuerzas tectónicas regionales [5]. Esta estructura empieza en la superficie y se extiende hasta una profundidad de $20 \mathrm{~km}$, en donde se une con la zona de subducción de la placa de Nazca. Esta característica es parte de una serie de zonas superficiales detectadas en diversos estudios, utilizando instrumentos pertenecientes a la Universidad de Tarapacá y mediante campañas de investigación sísmica realizadas por la Universidad de Chile [2, 6]. La característica más notoria es la dirección de toda la zona de fractura. Esta es una situación muy común en esta área y si representamos toda la sismicidad durante el año podemos ver que la estructura posee una geometría bien definida, como se puede apreciar en las Figuras 2.a y 2.b.

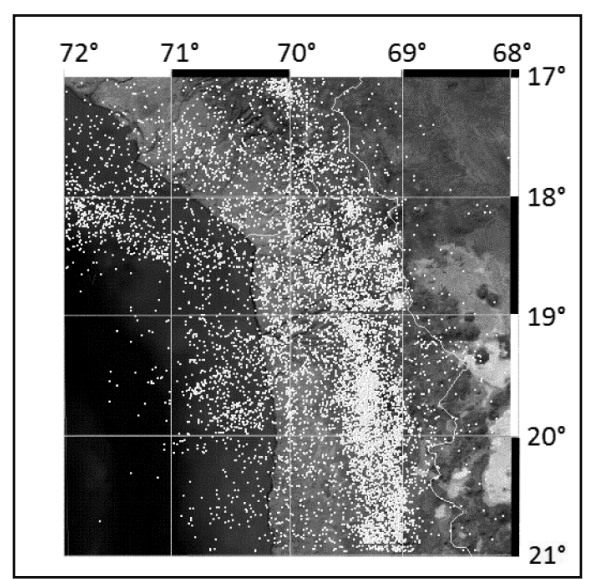

Figura 2.a. Epicentros de toda la sismicidad registrada el 2001.
En la Figura 2.b se ve la zona de subducción normal penetrando bajo la placa sudamericana, pero esta se proyecta hacia arriba en dirección a la superficie. Además se puede observar una zona secundaria, la que es la responsable de gran parte de la sismicidad que ocurre en el norte de Chile.

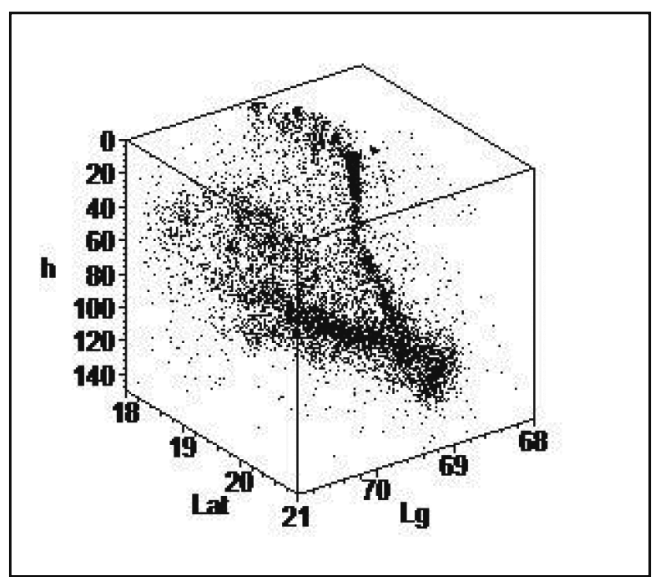

Figura 2.b. Sismicidad del 2001 vista en profundidad.

La parte superior de esta característica secundaria tiene una forma de arco siguiendo la del llamado Codo de Arica (Figura 3.a). En la Figura 3.b se muestra una vista vertical de la sismicidad en la zona en estudio.

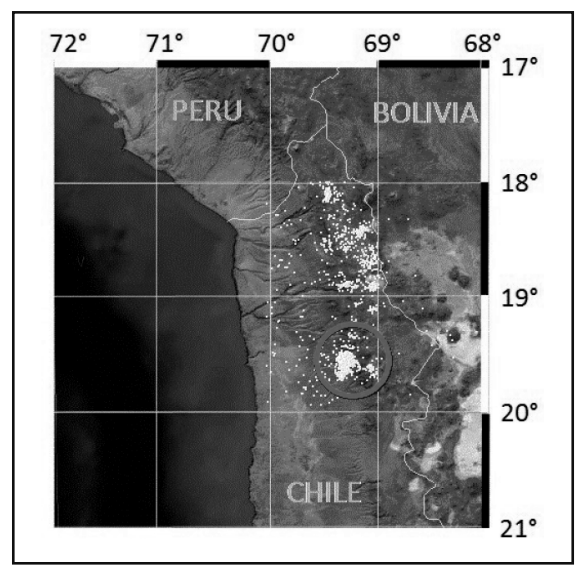

Figura 3.a. Todos los epicentros de sismicidad superficial donde el área estudiada está delimitada con un círculo. 


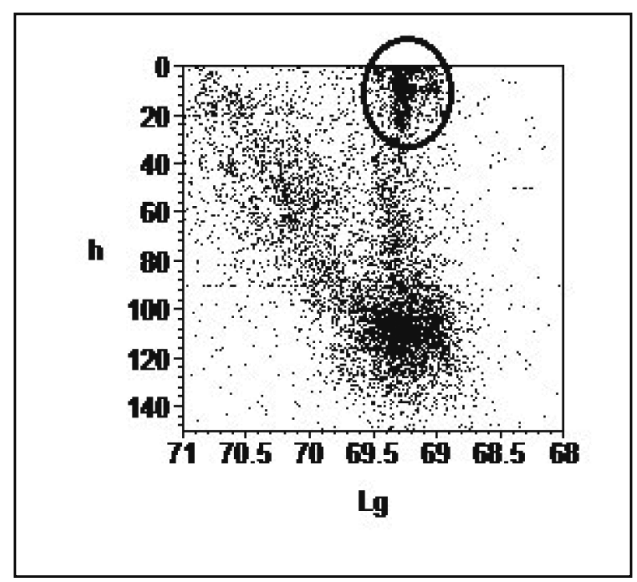

Figura 3.b. Sección longitudinal donde el círculo muestra la zona en estudio.

El evento ocurrido el 24 de julio y su sismicidad asociada fueron captados por medio del despliegue de la red de sismómetros que la Universidad de Tarapacá tiene instalada en la XV Región de Chile, la que consiste de 13 instrumentos de periodo corto ubicadas entre la ciudad de Arica y el norte de la ciudad de Iquique (Tabla 1). Esta red de sismómetros se llama "Red Sismológica Telemétrica de Arica" (Resiste-Arica) (Figura 4) y cuyas señales permitieron calcular las localizaciones hipocentrales con gran precisión debido a que la zona estudiada está incluida en el área de cobertura de la red [3].

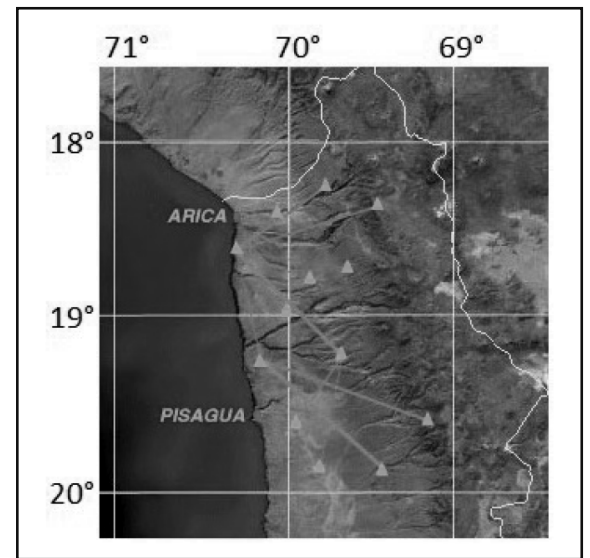

Figura 4. Los triángulos muestran la localización de los instrumentos de Resiste-Arica.

El margen de error al identificar eventos en una proyección horizontal y de profundidad es menor a 1,2 km para los 1.850 sismos ocurridos que fueron considerados en este estudio, pero no existe información acerca de la magnitud de ellos.

La importancia de la estructura mostrada aquí es lo suficientemente relevante para merecer más

Tabla 1. Estaciones de Resiste y sus características.

\begin{tabular}{|c|c|c|c|c|c|c|c|}
\hline Canal & Abrev. & Nombre & F(MHz) & Latitud & Longitud & $\begin{array}{l}\text { Alt. } \\
\text { (m) }\end{array}$ & $\begin{array}{l}\text { Dist. } \\
\text { (km) }\end{array}$ \\
\hline 0 & VIC & Víctor & 407.550 & $18^{\circ} 57.360^{\prime}$ & $70^{\circ} 03.910^{\prime}$ & 1235 & 40 \\
\hline 1 & GAR & Garza & 406.775 & $18^{\circ} 46.149^{\prime}$ & $69^{\circ} 55.273^{\prime}$ & 1530 & 45 \\
\hline 2 & CAR & Caricoya & 406.850 & $18^{\circ} 42.991^{\prime}$ & $69^{\circ} 39.469^{\prime}$ & 2880 & 71 \\
\hline 3 & CHA & Chapiquiña & 407.625 & $18^{\circ} 20.092^{\prime}$ & $69^{\circ} 30.035^{\prime}$ & 4470 & 92 \\
\hline 4 & ATA & Atajaña & 406.925 & $19^{\circ} 18.015^{\prime}$ & $70^{\circ} 08.284^{\prime}$ & 1490 & 84 \\
\hline 5 & SOT & Sotoca & 420.000 & $19^{\circ} 35.640^{\prime}$ & $69^{\circ} 12.890^{\prime}$ & 3416 & 172 \\
\hline 6 & TAR & Tarapacá & 420.100 & $19^{\circ} 54.700^{\prime}$ & $69^{\circ} 30.080^{\prime}$ & 1570 & 180 \\
\hline 7 & PUQ & Puquio & 470.000 & $18^{\circ} 14.765^{\prime}$ & $69^{\circ} 49.084^{\prime}$ & 3228 & 67 \\
\hline 8 & MIN & Miñimiñe & 406.300 & $19^{\circ} 11.806^{\prime}$ & $69^{\circ} 41.173^{\prime}$ & 1955 & 92 \\
\hline 9 & PAM & Pampa Ger & 420.050 & $19^{\circ} 52.530^{\prime}$ & $69^{\circ} 48.910^{\prime}$ & 1181 & 163 \\
\hline $\mathbf{A}$ & ZAP & Zapiga & 420.150 & $19^{\circ} 38.710^{\prime}$ & $69^{\circ} 58.580^{\prime}$ & 1260 & 130 \\
\hline B & POC & Poconchile & 406.450 & $18^{\circ} 27.294^{\prime}$ & $70^{\circ} 06.485^{\prime}$ & 1100 & 28 \\
\hline C & $\mathrm{CZ}$ & Camaraca & - & $18^{\circ} 37.003^{\prime}$ & $70^{\circ} 19.376^{\prime}$ & 915 & 14 \\
\hline D & CL & Camaraca & - & $18^{\circ} 37.003^{\prime}$ & $70^{\circ} 19.376^{\prime}$ & 915 & 14 \\
\hline $\mathbf{E}$ & CT & Camaraca & - & $18^{\circ} 37.003^{\prime}$ & $70^{\circ} 19.376^{\prime}$ & 915 & 14 \\
\hline $\mathbf{F}$ & GPS & (Tiempo) & - & - & - & - & - \\
\hline- & ARI & Arica & 451.600 & $18^{\circ} 29.430^{\prime}$ & $70^{\circ} 19.800^{\prime}$ & 61 & 0 \\
\hline
\end{tabular}


atención, ya que su geometría es complementaria a la de una subducción regional.

Teniendo en cuenta que las herramientas que se poseen y la teoría que se ha desarrollado en el estudio de la organización de la sismicidad, estas son de gran ayuda para entender el comportamiento de la zona de fractura y la posible predicción de eventos mayores. Se enfoca el problema del seguimiento de la organización de los eventos alrededor del epicentro del terremoto en estudio.

En este paper se trabajó con dimensiones multifractales, ya que con este método solo se requiere contar los eventos con una estimación mediana de la localización de los hipocentros, pues lo que se necesita es una idea de cómo está organizada la sismicidad, antes durante y después de la ocurrencia del evento principal.

El trabajo está organizado de la siguiente manera: En la sección dos, se utiliza un método multifractal y se determinan y subsanan algunos problemas de ejecución del cálculo que hay que tener en cuenta para no producir distorsión debido al número de eventos que se incluyen en los cálculos. Además, se da a conocer un modus operandi que proporciona buenos resultados al aislar los eventos organizados que son importantes para describir la ocurrencia del evento mayor y se muestran los resultados de la serie de índices calculados. En la sección tres se muestra el seguimiento espacial de los eventos, antes y después del evento principal y se correlaciona su evolución con el índice multifractal. En la sección cuatro se dan a conocer algunas conclusiones y otros estudios que merecen ser realizados en el futuro.

\section{CÁLCULO DEL ÍNDICE FRACTAL}

Para calcular el índice multifractal hemos elegido la siguiente definición:

$$
I_{q}=\frac{1}{q-1} \lim _{\varepsilon \rightarrow 0} \frac{\log _{10}\left(\sum_{i=1}^{N(\varepsilon)} p_{i}^{q}\right)}{\log _{10}(\varepsilon)}
$$

La ecuación (1) fue definida por Grassberger y Procaccia [4], donde $p_{i}=p_{i}(\varepsilon)$ es la probabilidad de ocupación de la caja $i$ de tamaño $\varepsilon$ y $q$ es un número real positivo o negativo. Esta definición toma en cuenta la distancia generalizada de orden $q$ entre puntos y puede ser vista como una generalización de orden $q$ de la dimensión de correlación. $\mathrm{N}$ es el número de cubos de tamaño $\varepsilon$.

En este caso se define una zona de cálculos alrededor del evento principal con las siguientes coordenadas: Latitud: $19^{\circ} 15^{\prime}$ a $19^{\circ} 45^{\prime}$; Longitud: 6900' a 69³0' y profundidad desde la superficie $(0 \mathrm{~km})$ hasta $30 \mathrm{~km}$.

Esta zona está rodeada por 13 estaciones, de las cuales dos de ellas están situadas en el área de estudio, además de 4 estaciones que están alrededor del borde, lo que permite obtener una muy buena determinación de la localización del hipocentro. Esta región fue subdividida en cubos de $\varepsilon=1,25 \mathrm{~km}$, un tamaño que es mayor que el margen de error al determinar los hipocentros, por lo que no se ve afectado el cálculo del índice fractal. Se utiliza el valor $q=2$, porque, como se puede apreciar en varios artículos [7-9], la forma de la curva del índice no es muy sensible al cambio con el valor de este parámetro.

El cálculo se lleva a cabo solo durante el año 2001, pero debido a que los temblores considerados son 1.850 , ellos permiten obtener buenos resultados en los cálculos y resultados estadísticos.

El procedimiento estándar es definir una ventana temporal para evitar el hecho de que el método es sensitivo al número de eventos [10]. De hecho, podemos ver que el análisis fractal es muy dependiente del número de casos que se consideran para calcular el índice en una zona determinada cuando el número total es menor de 500 eventos.

Debido a este valor umbral el índice fractal no es confiable cuando se calcula con un número de casos bajo 500. Sin embargo, si se cuenta con una curva de corrección, ella se puede utilizar para eliminar esa dependencia. Esto es importante porque no se desea perder la noción del tiempo en el que los eventos ocurren. Por tanto, se divide el año con el fin de calcular un índice representativo para cada semana. A lo largo del año se encuentran entonces 53 valores de índice, cada uno representando la organización promedio de la sismicidad en la región de estudio y para la semana correspondiente. Los resultados se muestran en la Figura 5. 
La semana " 0 " es la semana del evento principal del 24 de julio. Es posible observar que hay valores de índice elevados desde el evento principal hacia delante. A pesar de que hay un poco de actividad antes que ocurra el mencionado evento principal, es imposible hacer un comentario sobre la organización especial de sismicidad en este caso.

Como se puede observar en la Figura 5, hubo muy poca organización de la sismicidad en la semana antes de que ocurriera el evento principal. De hecho, el valor del índice en la semana anterior al evento es menor que el de las semanas $-15 \mathrm{y}-14$, cuando ningún evento importante fue detectado y ni siquiera se registró un sismo sensible en la zona.
Como se sabe que el índice es muy sensible al número de eventos, es posible que las características obtenidas estén provocadas por la asimetría en número que tienen los sismos antes y después del evento principal, por ello se procedió a eliminar este efecto.

Primero se grafica el índice versus el número de eventos y luego se obtiene un modelo que es logarítmico, como se puede apreciar en la Figura 6.

Al graficar los datos, podemos ver que el número de eventos (N) explica el $98 \%$ de la variación del índice tal como lo describe la Figura 7. Los parámetros del modelo son los siguientes: 4,13 $\pm 0,07$ para la pendiente y $0,3 \pm 0,1$ para el parámetro de posición.

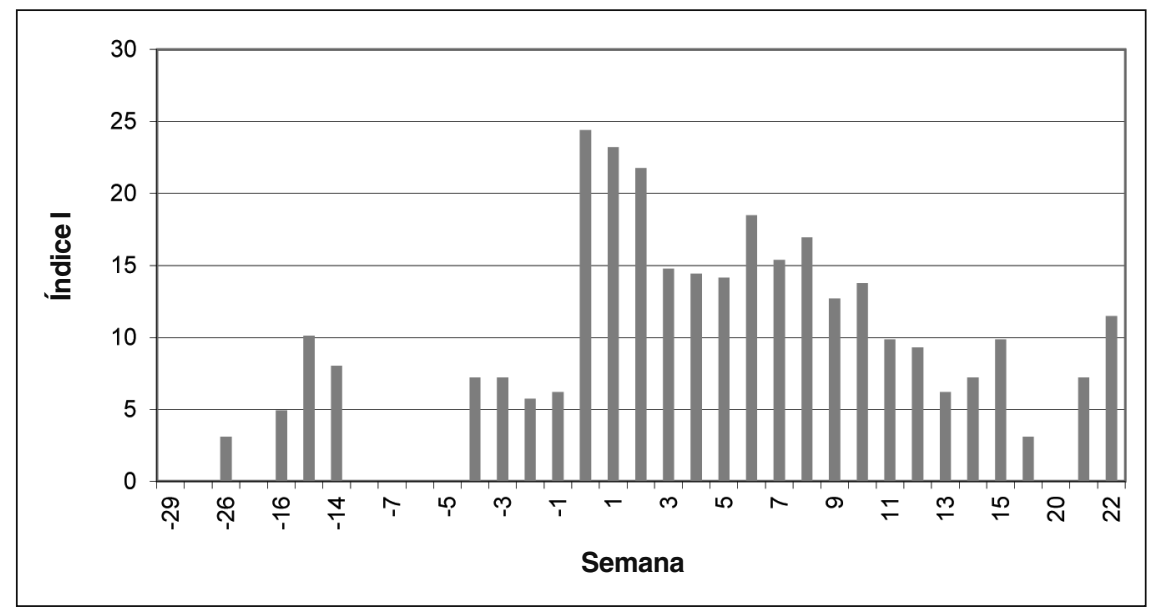

Figura 5. Índice multifractal para todo el año 2001.

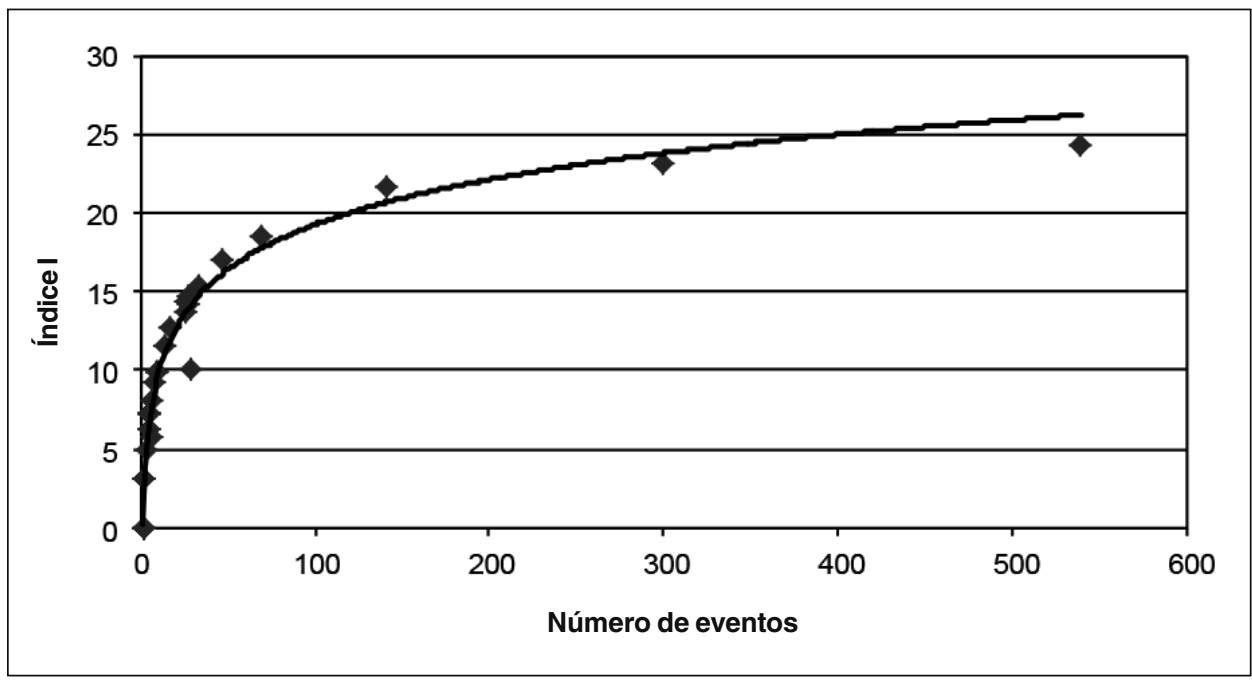

Figura 6. Índice multifractal versus el número de eventos. 
Al eliminar la dependencia en el número de eventos, obtenemos un nuevo índice corregido y así obtenemos las series descritas en la Figura 8.

Se puede ver ahora que claramente existen dos semanas previas al evento principal donde la organización fue muy importante, esto corresponde a las semanas $-15 \mathrm{y}-2$. Más aún, hay una importante organización en la semana del evento principal.

La eliminación de la dependencia del número de eventos demuestra ser muy útil, ya que ahora es posible ver la organización de los eventos que permanecían escondidos en el índice I. Entonces, es posible decir que a primera vista se podría obtener una señal de importancia en cuanto a la probabilidad de tener un evento mayor al observar este índice corregido. De hecho, podemos usar este procedimiento tan solo utilizando la información de la semana anterior, al punto que queremos corregir y aun así es posible obtener una organización más real de la sismicidad en la zona en relación con la que se observa sin corregir el índice, como se puede apreciar en la Figura 9.

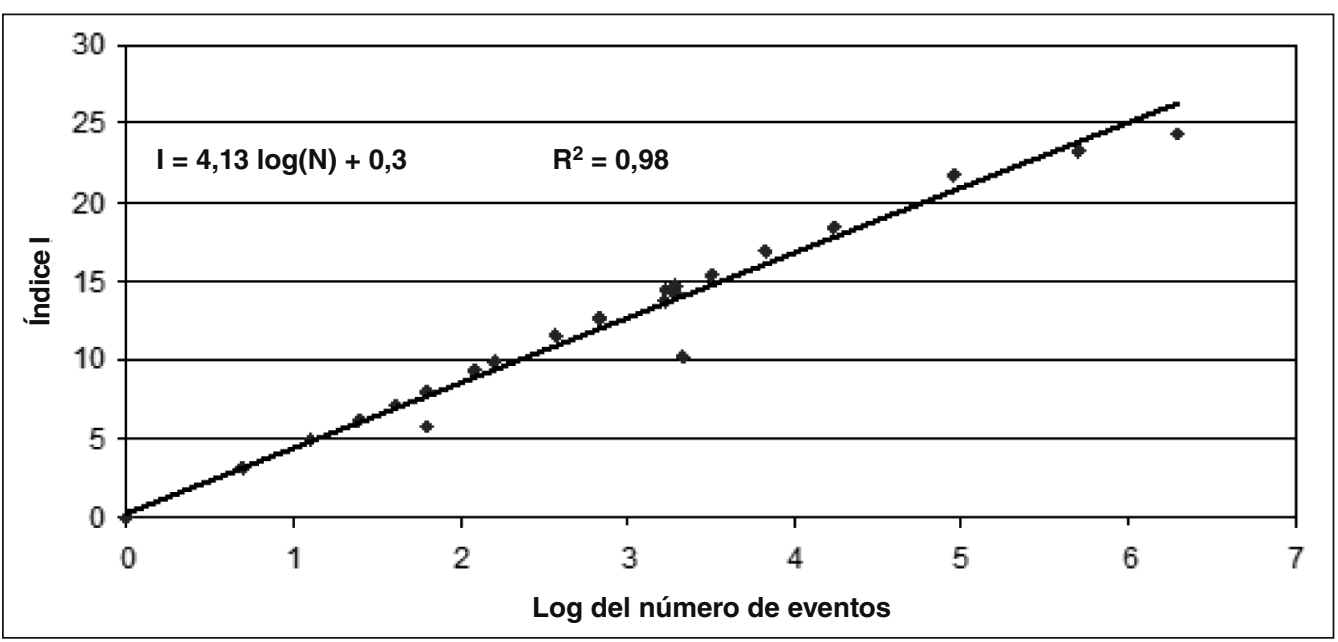

Figura 7. Modelo de la dependencia del índice con el número de eventos correspondientes a los datos reales.

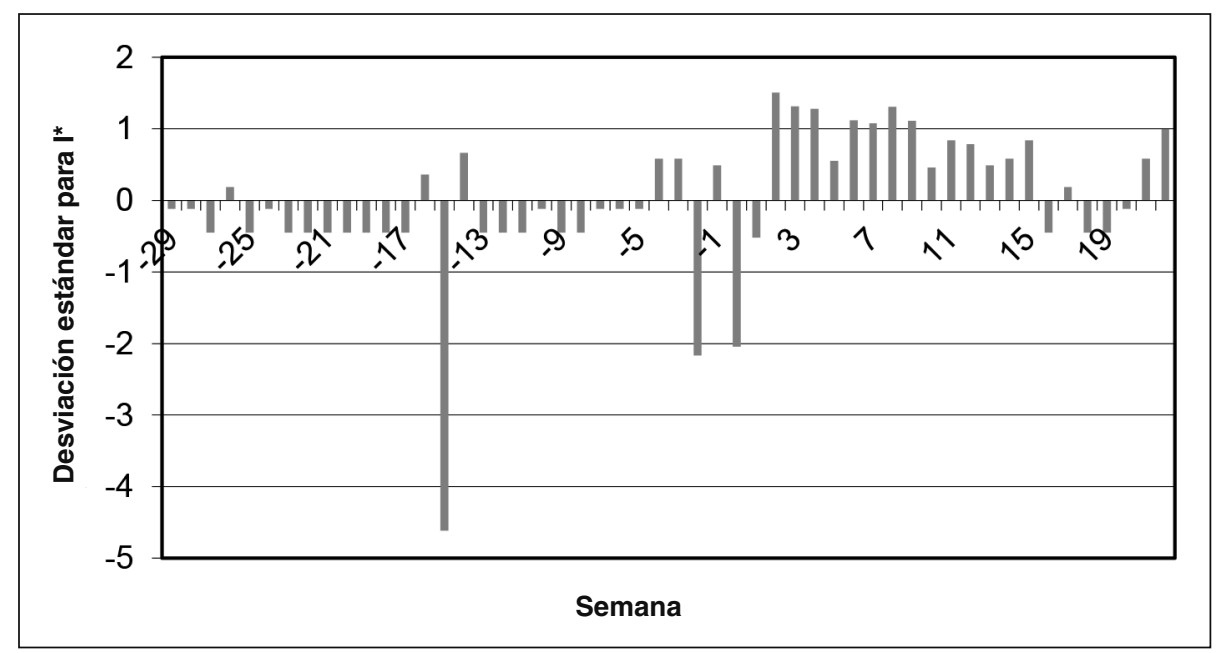

Figura 8. Índice corregido $I^{*}$. 


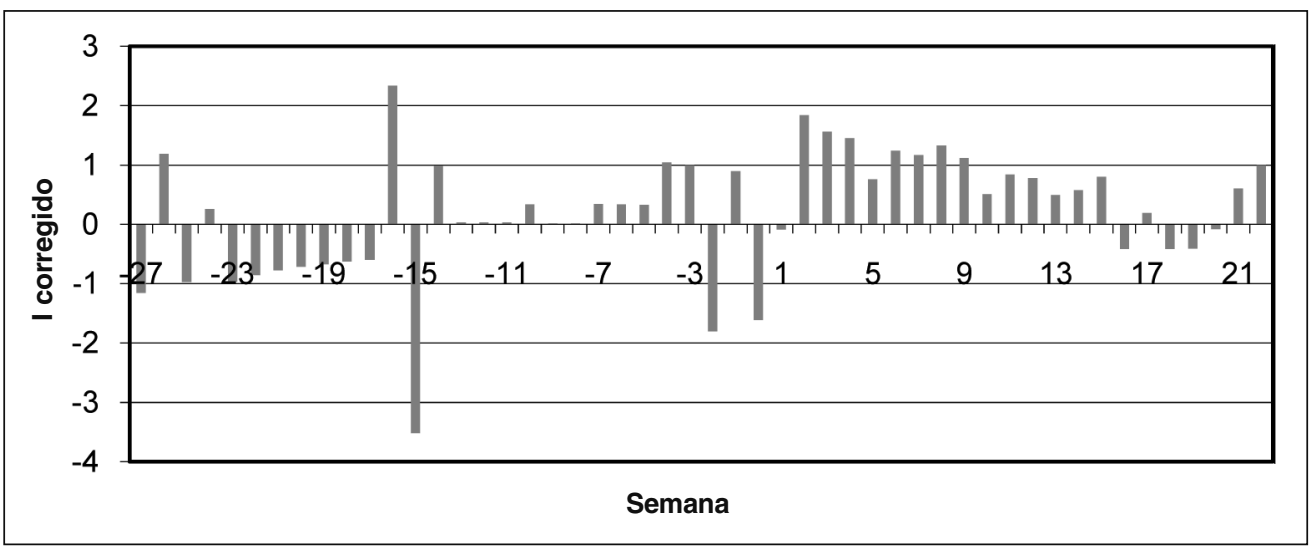

Figura 9. Índice I corregido con los datos de la semana previa a la graficada.

\section{SEGUIMIENTO ESPACIAL DE LA SISMICIDAD}

La Figura 9 muestra que en las semanas -15 y -2 hay una organización importante de la sismicidad. Es posible pensar, sin lugar a dudas, que la actividad de la semana -2 está muy relacionada con el evento principal.

En las Figuras 10 y 11 vemos representada la evolución de la sismicidad para las semanas -15 y -16 . Sin embargo, ¿qué pasa en la semana -15 ?

Se puede ver que hay una zona superficial de sismicidad que produce una señal de organización como podemos ver en el índice I* corregido (Figura 8).

La sismicidad desde la semana -2 hasta la semana +1 se representan en las Figuras 12 a la 15, para mostrar la evolución de la sismicidad dos semanas

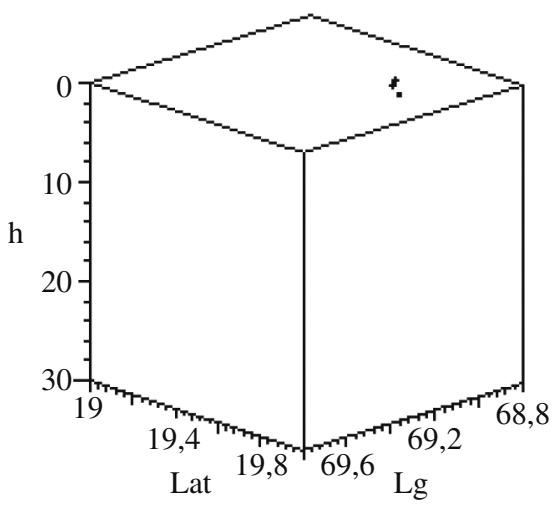

Figure 10. Sismicidad para la semana -16 .

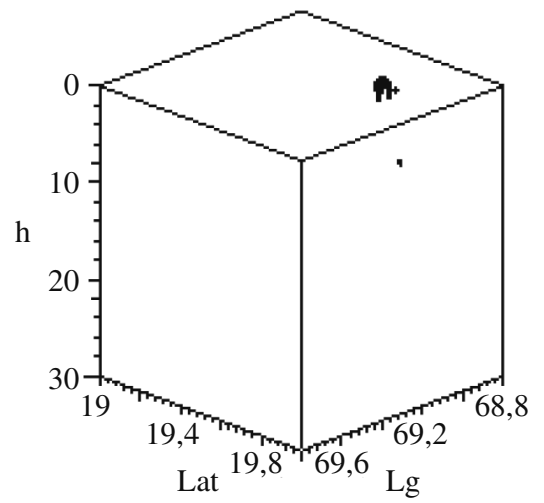

Figura 11. Sismicidad para la semana -15 .

antes del evento principal, durante la semana 0 y una después, donde claramente se ve la zona de fractura producida por el terremoto del 24 de julio del 2001.

También es posible ver que la zona de fractura de la semana -15 aún estaba presente cuando ocurrió el evento mayor, pero no se puede tomar el riesgo de hacer algún comentario acerca de su relación con el evento que se está estudiando.

\section{DISCUSIÓN Y PERSPECTIVAS}

En este artículo es posible ver que el índice multifractal es una importante herramienta para detectar la organización de la sismicidad cuando el efecto del número de eventos es eliminado. De hecho, podemos ver que el índice corregido puede entregarnos una pista significante de qué está 


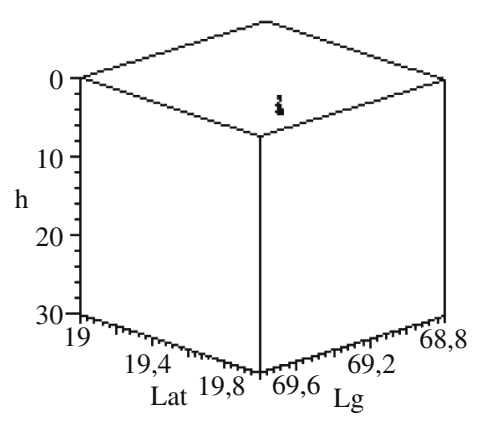

Figura 12. Sismicidad de la semana -2 .

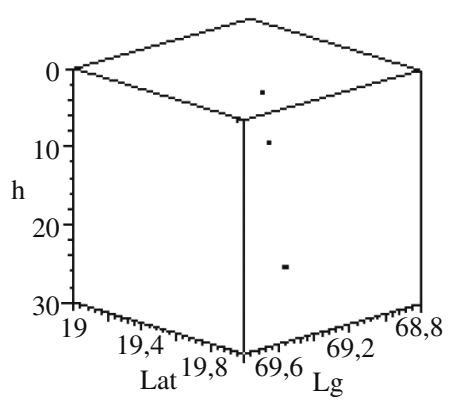

Figura 13. Sismicidad de la semana -1 .

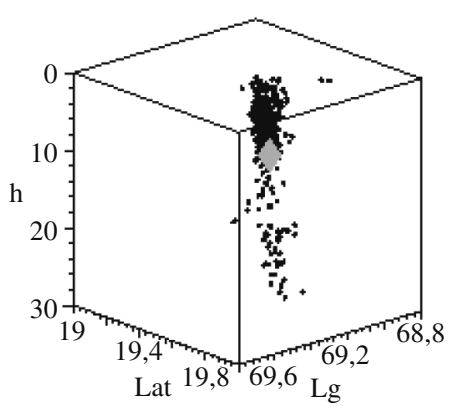

Figura 14. Sismicidad de la semana 0. El diamante muestra la ubicación del evento principal.

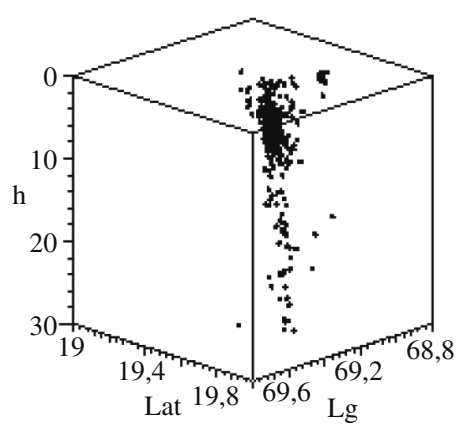

Figura 15. Sismicidad de la semana +1 . ocurriendo cuando se acerca un evento mayor. De cualquier forma, no significa que sea factible tener una herramienta de predicción, pero sí una noción aproximada de probabilidades de una ruptura mayor.

Se obtuvo un modelo empírico para eliminar el factor número de eventos, podría ser muy útil obtener un modelo más preciso incluyendo series más largas de datos desde la red local y para otras redes, determinando así si esta corrección es general o local.

Además, es importante destacar que no se consideró el mecanismo de ruptura de los 1.850 eventos, tampoco su magnitud, ya que no se cuenta con esa información y, por otro lado, es una información irrelevante para el método empleado.

\section{CONCLUSIONES}

Es factible construir un índice fractal que represente una herramienta útil para obtener una idea de la organización de la sismicidad en zonas definidas que son de gran interés por su riesgo sísmico. Esta herramienta también brinda cierto grado de predicción de eventos mayores y podría ser muy útil para estudios en ingeniería estructural.

Al construir este índice, la dependencia del número de los eventos debe ser tomada en cuenta. De esta forma, se ha obtenido un procedimiento empírico para corregir este efecto.

También es posible ver que la estructura donde se produjo el sismo principal en estudio está asociado a la zona principal de fractura de la región debido al mecanismo de subducción. Esta estructura es la responsable de la mayor parte de la sismicidad en el área y merece un estudio en profundidad.

Es importante destacar que este tipo de estudio no se ha realizado nunca en la zona norte de Chile, por lo que no es posible realizar algún tipo de comparación.

Asimismo, sería interesante considerar la posibilidad de llevar a cabo futuros estudios teniendo en mente la dependencia de la magnitud de los sismos y su relación con el índice fractal. 


\section{AGRADECIMIENTOS}

Este estudio fue patrocinado por el proyecto 472309 de la Universidad de Tarapacá.

\section{REFERENCIAS}

[1] D. Legrand. "Source parameters of the $M_{\mathrm{w}}=6.3$ Aroma crustal earthquake of July 24, 2001 (northern Chile), and its aftershock sequence". Journal of south American earth sciences. Vol. 24, Issue 1, pp. 58-68. 2007.

[2] M. Farías, R. Charrier, D. Comte, J. Martinod and G. Herail. "Deformación por flexuras en la precordilledra de la región de Aroma (19 $\left.25-19^{\circ} 45\right)$ : correlaciones con la sismicidad superficial e implicancias para el alzamiento del borde continental del Altiplano". Proceedings of $10^{\circ}$ Congreso Geológico Chileno. 2003.

[3] D. Comte, C. Dorbath, L. Dorbath, M. Faría, C. David, H. Haessler, B. Glass, E. Correa, I. Balmaceda, A. Cruz y L. Ruz. "Distribución temporal y en profundidad de las réplicas del sismo superficial de Aroma, norte de Chile del 24 de julio de 2001". $\mathrm{X}$ Congreso Geológico Chileno. Universidad de Concepción. Concepción, Chile. 2003.

[4] D. Comte, M. Bordier, R. Roroschek, C. David, J. Martinod, B. Glass, E. Correa, I. Balmaceda, L. Dorbath, H. Haessler, G. Herail, C. Meneses, M. Frogneux and A. Cruz. "Analysis of the 24 July 2001 Shallow Earthquake $\mathrm{Mw}=6.3$ Recorded in the Northern Chile Altiplano". American
Geophysical Union, Fall Meeting 2001, abstract \#S52A-0616. 2002

[5] C. David, D. Comte, L. Dorbath, H.J. Tavera, H. Haessler, M. Frogneux, L. Aaudin, G. Herail, G. Charrier, Reynaldo, B. Glass, I. Balmaceda, E. Correa, A. Cruz y L. Ruz. "Análisis sismotectónico en torno al codo de Arica: Resultados preliminares". X Congreso Geológico Chileno. Concepción, Chile, 6-10 Octubre 2003.

[6] D. Comte, C. Dorbath, R. Boroschek, L. Dorbath, B. Glass, E. Correa, C. Meneses, A. Cruz, H. Haessler and M. Frogneux. "Shallow Seismicity Around the Arica Bend in the Western Altiplano Piedmont". American Geophysical Union, Fall Meeting 2002, abstract \#S71C-1112. 2002.

[7] M. Eneva. "Monofractal of multifractal: a case study of spatial distribution of mining induced seismic activity". Nonlinear processes in Geophisycs. Vol. 1, pp. 182-190. 1994.

[8] P. Grassberger and I. Procaccia. "Measuring the Strangeness of Strange Attractors". Physica D: Nonlinear Phenomena. Vol. 9, Issue 1-2, pp. 189-208. 1983.

[9] D. Legrand, D. Villagómez, H. Yepes and A. Calahorrano. "Multifractal dimension and $b$ value analysis of the 1998-1999 Quito swarm related to Guagua Pichincha volcano activity, Ecuador". Journal of Geophysical Research. Vol. 109, B01307. 2004.

[10] P.N.S. Roy and Sankar K. Nath. "Precursory correlation dimensions for three great earthquakes". Current Science. Vol. 93, Issue 11. 2007. 